\title{
Modeling Phytoplankton Movement and Fitness in Lakes
}

\author{
Amy R. Lazarte \\ Biology Department, Reed College \\ lazartea@reed.edu
}

\author{
Samuel B. Fey \\ Biology Department, Reed College \\ feys@reed.edu
}

\author{
Anna Ritz \\ Biology Department, Reed College \\ aritz@reed.edu
}

\section{CCS CONCEPTS}

- Computing methodologies $\rightarrow$ Modeling and simulation; • Applied computing $\rightarrow$ Computational biology.

\section{KEYWORDS}

lake ecology, phytoplankton, fitness, movement

\section{INTRODUCTION}

The majority of organisms on Earth are ectothermic, and their body temperatures largely depend on the surrounding environmental conditions. Body temperature strongly affects fitness [2]. In a spatially variable environment, movement can allow an ectothermic organism to change their body temperature. This project explores body temperature and movement in the context of phytoplankton fitness within lake environments. By modelling movement strategies using lake thermal profile data, we infer the range of temperatures phytoplankton experience and the resulting effect on fitness.

\section{METHODS}

Temperature data was collected from Sparkling Lake in Vilas County, Wisconsin at intervals from .5 to $3 \mathrm{~m}$ throughout the lake at a frequency of one minute over a period of 26 years [1]. Phytoplankton fitness $w$ is a function of water temperature $T$ :

$$
w(T)=b_{1} e^{b_{2} T}-\left(d_{0}+\frac{b_{1} b_{2}}{d_{2}} e^{\left(b_{2}-d_{2}\right) T_{o p t}} e^{d_{2} T}\right)
$$

where $b_{1}$ represents birth rates at $0^{\circ} \mathrm{C}, b_{2}$ represents the exponential change in birth rate due to increased temperature, $d_{0}$ represents a temperature-independent mortality constant, $d_{1}$ and $d_{2}$ represent exponential changes to mortality rates due to increased temperature, and $T_{\text {opt }}$ represents the temperature at which growth rate is maximized [2]. We designed three movement algorithms to simulate patterns of phytoplankton movement across lake depths. Diel Vertical Migration (DVM) represents the daily pattern of movement observed in certain species of phytoplankton. Phytoplankton move upwards during the day and downwards at night. Two phytoplankton speeds were implemented. Random Walk (RW) randomly samples depths independent of the distance traveled. Directional Random Walk (DRW) randomly samples depths confined to the local neighborhood.

Permission to make digital or hard copies of part or all of this work for personal or classroom use is granted without fee provided that copies are not made or distributed for profit or commercial advantage and that copies bear this notice and the full citation on the first page. Copyrights for third-party components of this work must be honored. For all other uses, contact the Owner/Author.

$A C M-B C B$ '19, September 7-10, 2019, Niagara Falls, NY, USA

(C) 2019 Copyright is held by the owner/author(s).

ACM ISBN 978-1-4503-6666-3/19/09.

https://doi.org/10.1145/3307339.3343236

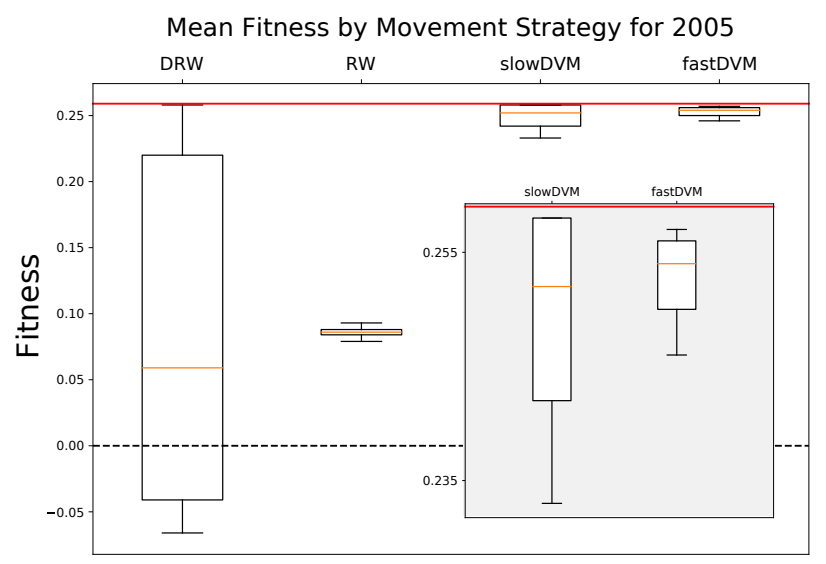

Figure 1: Mean body fitness across 1000 runs of all five movement strategies over the 2005 warm season. The solid red line indicates the highest possible mean fitness. The inset shows two DVM speeds.

\section{RESULTS AND DISCUSSION}

The two speeds of diel vertical migration both achieve mean fitness nearly as high as the maximum fitness (Figure 1). During the summer, the day period is longer than the night period. This means that the phytoplankton can swim further upwards than they are able to swim downwards in the night. Regardless of starting position, phytoplankton will eventually reach the warmer temperatures at the lake's surface and achieve higher fitness. However, slower phytoplankton have a greater variance in fitness because they take longer to reach the surface (figure inset). The random walk movement pattern demonstrates the average body temperature experienced over space and time. The large variance seen in the directional random walk demonstrates the wide spread of fitness achieved at varying depths in the lake. Together, these movement patterns allow us to quantify potential thermal fluctuations phytoplankton experience as they move throughout their environment, and identify how different patterns of movement influence fitness over time.

Acknowledgements. This work was supported by the Stafford Research Fellowship Fund and NSF awards ABI-1750981 (to AR) and DEB-1856415 (to SBF). We thank Colin Kremer for fitness functions and parameters.

\section{REFERENCES}

[1] J. J. Magnuson et al. 2013. North Temperate Lakes LTER: High Frequency Water Temperature Data - Sparkling Lake Raft 1989 - current. (2013). http://lter. limnology.wisc.edu [Online; accessed 10-September-2018].

[2] Mridul K. Thomas et al. 2017. Temperature-nutrient interactions exacerbate sensitivity to warming in phytoplankton. Global Change Biology 23, 8 (2017), 3269-3280. 